\title{
Let-Polymorphism and Eager Type Schemes
}

\author{
Chuck Liang \\ Department of Computer Science \\ Frostburg State University \\ Frostburg, MD 21532, USA
}

\begin{abstract}
This paper presents an algorithm for polymorphic type inference involving the let construct of ML in the context of higher order abstract syntax. It avoids the polymorphic closure operation of the algorithm $W$ of Damas and Milner by using a uniform treatment of type variables at the meta-level. The basic technique of the algorithm facilitates the declarative formulation of type inference as goal-directed proof-search in a logical frameworks setting.
\end{abstract}

\section{Introduction}

Formulations and algorithms for the assignment of principal types to untyped $\lambda$ terms have long existed before Damas and Milner [2] extended it to involve the polymorphic let construct of functional programming languages (ML). They formulated a declarative, proof-theoretic calculus for the ML type system, given here in Figure 1. Unfortunately, this calculus does not by itself lead directly to an inference algorithm that yields principal type schemes. For this purpose the algorithm " $W$ " was given. Algorithm $W$ requires the polymorphic closure operation called gen (or close) in typing let-expressions. Together with the unification algorithm, this operation ensures maximal generality of the type scheme for the locally-bound term in let expressions. With respect to the original Damas-Milner calculus, gen effectively represents a forward-chaining step. Its introduction obscured the relationship between the declarative type system and the type-inferencing process (and a proof of completeness for $W$ was not offered until Damas' thesis). In particular, we shall show that algorithm $W$ entails an unnatural treatment of free and bound type variables. A common practice is to bypass let by replacing let $x=M$ in $N$ with $N[M / x]$. This replacement, however, is unsatisfactory because it leads to redundant inferences. The problem with gen becomes especially acute when one tries to formulate type inference in the context of logical frameworks, which are meta-theoretic environments designed to support the syntax of object-level theories in a natural manner. It is advantageous to formulate principal type inference, in such frameworks, as deterministic proof search (in the manner of logic programming). Numerous attempts have been made along these lines (eg, Pfenning [3]), all of which were limited by complications involving the gen operation. We aim to provide an alternative to algorithm $W$ (more specifically to using polymorphic closure) which will facilitate the formulation of polymorphic typing in declarative settings such as ELF [8], Coq [4], Isabelle [16], $\lambda$ Prolog [14], among others. 


$\begin{array}{lc}\text { Proj } & \overline{H \vdash x: T, \quad x: T \in H} \\ \text { abs } & \frac{H, x: s \vdash M: t}{H \vdash \lambda x \cdot M: s \rightarrow t} \\ \text { app } & \frac{H \vdash M: s \rightarrow t \quad H \vdash N: s}{H \vdash(M N): t} \\ \text { let } & \frac{H \vdash M: S \quad H, x: S \vdash N: t}{H \vdash \operatorname{let} x=M \text { in } N: t} \\ \text { I-Intro } & \frac{H \vdash M: T \quad(a \text { not free in } H)}{H \vdash M: \Pi a . T} \\ M \text {-Elim } & \frac{H \vdash M: \Pi a . T}{H \vdash M: T[s / a]}\end{array}$

( $s, t$ represent unquantified types; $S, T$ represent arbitrary type schemes)

Fig. 1. The Damas-Milner Calculus [2]

In this paper we present an algorithm for type inference that avoids the use of the gen operation. This algorithm will be presented in a meta-language based on the simply typed $\lambda$-Calculus, which is also the language used in several logical frameworks and logic programming interpreters. In particular, we shall show how the proper scoping of type variables can be formulated using $\lambda$-abstractions and how the polymorphism of types can be implemented with the simple rule of $\alpha$-conversion.

This paper is organized as follows. In Section 2 we discuss and present the algorithm. In Section 3 we give some sample type inferences using the algorithm. Sketches of correctness proofs are given in Section 4. We then describe how the algorithm is implemented in a declarative setting in Section 5. In Section 6 we discuss the significance of our technique with respect to related research in conjunctive typing disciplines, including those of Leivant [12], Appel and Shao [1], among others.

\section{Free, Bound, and "Fugitive" Variables}

Technically, the algorithm $W$ infers types, and not type schemes. Let $\overline{v_{m}}$ denote $v_{1}, \ldots, v_{m}$. Whenever a typing assumption $f: \Pi \bar{v}_{m} . t$ is used, a "copy" of the type $t\left[\overline{x_{m}} / \overline{v_{m}}\right]$ is created using a set of new free variables $\overline{x_{m}}$. This occurs uniformly except in the let case, when type scheme inference takes place in the form of applying gen. The technique we use approaches type inference from the opposite direction. Here type scheme inference is the default. In other words, we shall always try to keep type variables $\Pi$-quantified as much as possible. If the typing of a compound expression e requires two instances of a type scheme $I I \bar{v} . t$, this is made possible by appending two copies of the quantifier prefix to 
yield $\Pi \overline{v_{m}} \Pi \overline{v_{m}^{\prime}} \cdot s$, where $s$ is the type of $e$. New free variables are uniformly replaced by new $I I$-bound variables. Typing conflicts are resolved post-hoc to prevent over-generalization.

We now present the algorithm in detail. The algorithm takes advantage of the fact that in practice, only closed type environments are needed. With closed environments, all free type variables that are dynamically introduced during the type inferencing process can be safely discharged ( $\Pi$-quantified) upon successful completion of the process. As in Damas-Milner, only in the inductive proofs of correctness need we be concerned with the more general case of open environments.

Define an extended type environment $H_{e}$ to be a mapping from program (or term) variables $x$ to structures of the form $A \overline{v_{m}} .(\sigma, t)$, which we shall refer to as eager type schemes. Here, $\sigma$ is a substitution on type variables and $t$ is a type such that $\sigma(t)=t$. The meta-level binding construct $A$ quantifies over the type variables $\overline{v_{m}}$, which may occur anywhere in the substitution-type pair $(\sigma, t)$. The intuitive meaning of this mapping is that $x$ maps to the potential type scheme $\Pi \overline{v_{m}} . t$ if the substitution $\sigma$ is applied to the current type environment. The algorithm, which we shall call $W_{I I}$, is given in Figure 2.

$W_{\Pi}\left(H_{e} ; x\right)=H_{e}(x)$, for program variable $x$.

$W_{I I}\left(H_{\epsilon} ; \lambda x . M\right)=$ let $a$ be a new type variable, and let

$$
\begin{gathered}
W_{I I}\left(H_{e}, x \mapsto(\emptyset, a) ; M\right)=_{\alpha} \Lambda \overline{v_{n}} .(\sigma, t) . \\
\quad \text { Return } \Lambda a \Lambda \overline{v_{n}} .(\sigma, \sigma(a \rightarrow t)) .
\end{gathered}
$$

$W_{I I}\left(H_{e} ;(M N)\right)=$ let

$$
W_{I I}\left(H_{e} ; M\right)={ }_{\alpha} \Lambda \overline{v_{n}} .\left(\sigma_{1}, t_{1}\right) \text {, and } W_{\Pi}\left(H_{e} ; N\right)={ }_{\alpha} \Lambda \overline{u_{m}} \cdot\left(\sigma_{2}, t_{2}\right)
$$

such that the bound variables $\overline{u_{m}}$ are distinct from $\overline{v_{n}}$. For a new type variable $b$, let $\theta$ be the most general unifier of $t_{1}$ and $t_{2} \rightarrow b$. Let $\sigma=$ $\operatorname{join}\left(\theta, \sigma_{2}, \sigma_{1}\right)$.

$$
\text { Return } \Lambda b \Lambda \overline{u_{m}} \Lambda \overline{v_{n}} .(\sigma, \sigma(b)) .
$$

$$
\begin{aligned}
& W_{\Pi}\left(H_{e} ; \text { let } x=M \text { in } N\right)=\text { let } \\
& \qquad W_{\Pi}\left(H_{\epsilon} ; M\right)={ }_{\alpha} \Lambda \overline{v_{n}} .\left(\sigma_{1}, t_{1}\right), \text { and } \\
& W_{\Pi}\left(H_{e},\left(x \mapsto \Lambda \overline{v_{n}} \cdot\left(\sigma_{1}, t_{1}\right)\right) ; N\right)==_{\alpha} \Lambda \overline{u_{m}} \cdot\left(\sigma_{2}, t_{2}\right)
\end{aligned}
$$

such that $\overline{u_{m}}$ are distinct from $\overline{v_{n}}$. Let $\sigma=j$ join $\left(\sigma_{2}, \sigma_{1}\right)$.

$$
\text { Return } \Lambda \overline{u_{m}} \Lambda \overline{v_{n}} .\left(\sigma, \sigma\left(t_{2}\right)\right) \text {. }
$$

Fig. 2. Algorithm $W_{I I}$ 
For an extended type environment $H_{e}$ and a program expression $M$, $W_{\Pi}\left(H_{e} ; M\right)$ returns a structure $\Lambda \bar{v}_{m} \cdot(\sigma, t)$. Let represent the empty (or identity) substitution. We use only idempotent substitutions $(\theta \circ \theta=\theta)$. The operation join is borrowed from Leivant [12]. Given substitutions $S_{1}, \ldots, S_{n}$, $\operatorname{join}\left(S_{1}, \ldots, S_{n}\right)=R$ such that for each $S_{i}$ in $S_{1}, \ldots, S_{n}$ there is a substitution $P_{i}$ such that $P_{i} \circ S_{i}=R$. Furthermore, if $R^{\prime}$ also satisfies this property then there is a substitution $P$ such that $P \circ R=R^{\prime}$. That is, $\operatorname{join}\left(S_{1}, \ldots, S_{n}\right)$ is the most general common instance of $S_{1}, \ldots, S_{n}$ (if it exists). The join operation can be implemented using the standard unification algorithm.

The use of $\alpha$-equivalence $\left(=_{\alpha}\right)$ in the definition of the algorithm is appropriate since the $\Lambda$ binder is conveniently represented by $\lambda$-abstraction of the $\lambda$-calculus. This amounts to using higher-order abstract syntax [17] for our presentation. We use " $A$ " to distinguish it from the " $\lambda$ " used in program expressions.

To explain how this algorithm is used relative to a regular (non-extended) type environment, we define the following:

Definition 1 (Base Extension). Given a type environment $H$, let $H \uparrow$ represent the extended type environment that includes $\left(x \mapsto \Lambda \overline{v_{m}} .(\emptyset, t)\right)$ for each $\left(x: \Pi \overline{v_{m}}, t\right)$ in $H$.

For a closed type environment $H$, if $W_{\Pi}(H \uparrow ; M)$ succeeds with $\Lambda \overline{v_{m}} \cdot(\sigma, t)$ then it will be the case that $(\sigma, t)$ contains no free variables. We can then conclude that $H \vdash M: \Pi \overline{v_{m}} . t$.

The critical point in $W_{I I}$ where "free variables" are dynamically introduced into an environment occurs in the typing of a $\lambda$-expression $\lambda x . M$. Here $x$ is assumed to have type $a$, where $a$ is a new type variable. This variable is free only in the dynamic, temporary environment. It will be captured by $\Lambda$-abstraction when the top-level type scheme of $\lambda x . M$ is constructed. We will call the free variables introduced for $\lambda$-bindings fugitive variables.

The algorithm $W$ of Damas-Milner requires the prolific generation of new free variables. We observe, however, that if the initial environment is closed then all dynamically generated free variables that can not be immediately quantified are those that are unified with fugitive variables. But since the fugitive variables will also be quantifiable eventually, any new variable that occurs in a substitution for them will also be quantifiable eventually. In algorithm $W_{\Pi}$, all new variables generated from discharging (an instance of) a typing assumption are immediately quantified. As a consequence, some invalid expressions will appear "momentarily typable." The join operation, however, will reveal any inconsistencies in the substitutions and reject untypable expressions. We illustrate this technique of "eager quantification, delayed resolution" with three examples.

\section{Sample Inferences}

Assume the type environment $H$ contains the assignment $f: \Pi v . v \rightarrow v$. Consider typing the expression $\lambda x .(f x)$. First we augment the extended environment $H \uparrow$ 
with $x \mapsto(\emptyset, a)$ for a new fugitive variable $a$. In typing $(f x)$, we unify $v \rightarrow v$ with $a \rightarrow b$ for some new variable $b$. Thus

$$
W_{I I}(H \uparrow, x \mapsto(\emptyset, a) ;(f x))={ }_{\alpha} \Lambda b \Lambda v \cdot([v / a, v / b], v) .
$$

The accompanying substitution $[v / a, v / b]$ is then applied to $a \rightarrow v$, and the fugitive $a$ is "captured," yielding $A a A b \Lambda v \cdot([v / a, v / b],(v \rightarrow v)$. We can therefore conclude that $H \vdash \lambda x .(f x): \Pi a \Pi b \Pi v . v \rightarrow v$.

Now consider let $x=\lambda y . y$ in $(x x)$. First, $\lambda y . y$ is inferred as having the eager type scheme $A v .(\emptyset, v \rightarrow v)$. Then $x$ is assumed to map to this eager scheme. For $(x x)$, the type of $x$ is inferred twice as $A v \cdot(\emptyset, v \rightarrow v)$ and $\Lambda w \cdot(\emptyset, w \rightarrow w)$. With a new variable $b,(w \rightarrow w) \rightarrow b$ is unified with $v \rightarrow v$, yielding the substitution $[w \rightarrow w / b, w \rightarrow w / v]$. This substitution can be trivially joined with the two instances of the empty substitution inferred above. Thus calling $W_{I I}$ on $(x x)$ will return the structure

$$
\Lambda b \Lambda w \Lambda v \cdot([w \rightarrow w / b, w \rightarrow w / v], w \rightarrow w),
$$

and since the substitution returned joins immediately with the empty substitution in $\Lambda v .(\emptyset, v \rightarrow v)$, we can conclude that let $x=\lambda y . y$ in $(x x)$ has type $\Pi w . w \rightarrow w$ (eliminating the vacuous quantifiers this time for convenience; we may also implement this elimination as an optimization). The key observation here is that a type scheme is always inferred, thereby eliminating the need for the gen operation.

For the final example, assume the program variable $p$ has type $I v \cdot v \rightarrow v \rightarrow v$. Consider the untypable expression $\lambda y$. (let $x=(p y)$ in $(x x))$. For the top level $\lambda$-abstraction, a new fugitive variable $a$ is assumed as the type for $y$. In the let expression, $(p y)$ can be inferred as having the structure $A b A v \cdot([v / a,(v \rightarrow$ $v) / b], v \rightarrow v)$. The program variable $x$ is then assumed to map to this structure in the updated extended environment. Typing $(x x)$ will again produce two individual copies of this structure:

$$
\Lambda b . \Lambda v \cdot([v / a,(v \rightarrow v) / b], v \rightarrow v) \text {, and } \Lambda b_{2} . \Lambda w \cdot\left(\left[w / a,(w \rightarrow w) / b_{2}\right], w \rightarrow w\right) .
$$

Another type variable $b_{3}$ is introduced, and $(w \rightarrow w) \rightarrow b_{3}$ is unified with $v \rightarrow v$, resulting in the substitution $\left[(w \rightarrow w) / v,(w \rightarrow w) / b_{3}\right]$. But this substitution can not be joined with the two substitutions from the individual recursive inferences for $y:[v / a,(v \rightarrow v) / b]$, and $\left[w / a,(w \rightarrow w) / b_{2}\right]$. The variable $a$ can not have both $w \rightarrow w$ and $w$ (or both $v \rightarrow v$ and $v$ ) as instances.

Notice that although a fugitive $a$ is a (dynamically) free variable, it can be substituted by a $(\Lambda)$ bound variable, as when $a$ was substituted by the $\Lambda$ bound variable $v$ in the third example. Once a variable is bound, "copies can be made", and thus two instances of $v, v$ and $w$, were created. Type inference was allowed to continue where in algorithm $W$ it would have failed: $v$ was unified with $w \rightarrow w$. This "eager inference," however, was invalidated when the substitutions were joined, revealing that $v / a$ and $w / a$ are inconsistent if $v=w \rightarrow w$. In case these substitutions can be successfully joined, then these variables ( $v$ and $w$ ) can remain rightfully quantified, since the final type scheme returned will quantify 
over all fugitive variables. Because we need to keep track of which bound variables are in fact "eagerly" quantified, the join operation must replace the composition of substitutions as used in algorithm $W$. That is, we need to "memorize" the various substitutions for the fugitive variables in the form of extended type environments.

\section{Correctness Proofs}

This section addresses the major components required to show soundness and in particular completeness of $W_{\Pi}$ with respect to principal type schemes for the Damas-Milner typing discipline. As a consequence we also show how to extend the algorithm to accommodate open type environments in general.

With respect to a structure $\Lambda \bar{v}_{m} .(\sigma, t)$, we say that a bound variable $v_{i}$ is innocent if for some free variable $a, \sigma(a)=t$ such that $v_{i}$ occurs in $t$. That is, innocent variables are variables that were $A$-bound prematurely, and should be freed if $a$ occurs in the environment.

Definition 2 (Base Compression). Given an extended type environment $H_{\varepsilon}$ of the form

$$
\left\{x_{1} \mapsto \overline{\Lambda v_{n_{1}}^{1}}\left(\sigma_{1}, t_{1}\right), \ldots, x_{m} \mapsto\left(\Lambda \overline{v_{n_{m}}^{m}} \cdot\left(\sigma_{m}, t_{m}\right)\right\} .\right.
$$

Assume that all variables $v_{j_{k}}^{i}$ are distinct. Let $\delta=j \operatorname{oin}\left(\sigma_{1}, \ldots, \sigma_{m}\right)$. Let $\overline{u_{k}}$ be all the variables in $\delta$ that are innocent. Let $\overline{w_{l}}$ be all the variables $\overline{v_{n_{1}}^{1}}, \ldots, \overline{v_{n_{m}}^{m}}$ minus $\overline{u_{\bar{k}}}$. Define $\boldsymbol{H}_{e} \downarrow=(\delta, H)$ where $H$ is the type environment

$$
\left\{x_{1}: \Pi \overline{w_{l}} \delta\left(t_{1}\right), \ldots, x_{m}: \Pi \overline{w_{l}} . \delta\left(t_{m}\right)\right\} .
$$

For a type environment $H$, clearly $H \uparrow \downarrow=(\emptyset, H)$.

Theorem 3. Given an extended type environment $H_{e}$ and a program expression $M$, assume $W_{\Pi}\left(H_{e} ; M\right)=\Lambda \overline{v_{m}} \cdot(\sigma, t)$. If $\left[H_{e}, y \mapsto \Lambda \overline{v_{m}} .(\sigma, t)\right] \downarrow=(\delta, H)$ for some new "dummy" variable $y$, then $H \vdash M: H(y)$.

Proof. By structural induction on $M$, appealing to properties of the join operation.

We forgo the details of the soundness proof in favor of completeness. The following corollary establishes soundness for closed type environments.

Corollary 4. (Soundness of $W_{I I}$ ) Given a closed type environment $H$ and a term $M, W_{I}(H \uparrow ; M)=\Lambda \overline{v_{m}} \cdot(\sigma, t)$
implies $H \vdash M: \Pi \overline{v_{m}} . t$.

The structure of the (syntactic) completeness proof is similar to other such proofs including those of Leivant [12]. The main contribution here is our let case. Since there is no gen operation, in the proof of the let case the inductive 
hypothesis can be used directly. Most of the detailed proof deals with ordinary algebraic manipulations of the various substitutions. We define the generic application of a substitution $G$ to a type scheme $\Pi \overline{v_{m}} \cdot t$ as $G\left[\Pi \overline{v_{m}} \cdot t\right]=\Pi \overline{v_{m}} . G(t)$. That is, generic application can replace bound variables as well as free variables. For every "generic instance" (in the sense of Damas-Milner [2]) $\sigma^{\prime}$ of $\sigma$ there is a substitution $G$ such that $G[\sigma]=\sigma^{\prime}$ (modulo some vacuous $\Pi$ quantifiers). Because the $\downarrow$ operation breaks quantifiers, the completeness theorem must be stated using generic applications of substitutions. In the theorem below, we assume that all variables (free and bound) in $H_{e}$ are distinct.

Theorem 5. Assume for the extended type environment $H_{e}, H_{e} \downarrow$ exists and is equal to $(\delta, H)$. Assume $S[H] \vdash M: T$ for substitution $S$, term $M$ and type scheme $T$. Then $W_{\Pi}\left(H_{e} ; M\right)=\Lambda \overline{v_{m}} \cdot(\sigma, t)$. For a new term variable $y$, let $\left[H_{e}, y \mapsto \Lambda \overline{v_{m}} \cdot(\sigma, t)\right] \downarrow=\left(\delta^{\prime}, H^{\prime}\right)$, let $\theta \circ \delta=\delta^{\prime},{ }^{1}$ and let $H^{\prime}(y)=\Pi \overline{w_{l}} \cdot t^{\prime}$. It also holds that there exists a substitution $\rho$ such that $\rho \circ \theta=S$ and $\rho\left[\Pi \overline{w_{l}} \cdot t^{\prime}\right]=T$.

Proof. By induction on the height of derivations. For the inductive basis if $x$ : $\Pi \overline{w_{l}}, t_{0} \in H$ then $x \mapsto \Lambda \bar{v}_{m} .(\sigma, t) \in H_{e}$ for some $\sigma$ and $t$, and $W_{\Pi}\left(H_{e} ; x\right)=$ $\Lambda \overline{v_{m}} .(\sigma, t)$. Here, $\delta^{\prime}=\delta$. We set $\rho=S$ in this case and the result follows. The $\Pi$-Elim case is trivial. The $\Pi$-Intro case also follows easily since all variables not free in $H_{e}$ are always $\Lambda$-bound. The abs and app cases can be shown by rewritting the inference rules into more general forms:

$$
\frac{S[H], x: S[a] \vdash M: S[c]}{S[H] \vdash \lambda x \cdot M: S[a \rightarrow c]} a b s \quad \frac{S[H] \vdash M: S[r \rightarrow b] \quad S[H] \vdash N: S[r]}{S[H] \vdash(M N): S[b]} a p p
$$

where $a, c, r$ and $b$ are distinct type variables not appearing elsewhere.

We concentrate on the let case. Let $H_{e} \downarrow=(\delta, H)$. A let rule-application can be written in the form

$$
\frac{S[H] \vdash M: \xi \quad S[H], x: \xi \vdash N: T}{S[H] \vdash \text { let } x=M \text { in } N: T} \text { let }
$$

where $\xi$ is some type scheme. Then by inductive hypothesis, $W_{I I}\left(H_{e} ; M\right)=$ $\Lambda \overline{v_{m}} \cdot\left(\sigma_{1}, t_{1}\right)$ such that $\left[H_{e}, y \mapsto \Lambda \overline{v_{m}} \cdot\left(\sigma_{1}, t_{1}\right)\right] \downarrow=\left(\delta_{1}, H_{1}\right)$. Let $H_{1}(y)=\Pi \overline{w_{l}} \cdot t$ and $\theta \circ \delta=\delta_{1}$. There is also a substitution $\rho_{1}$ such that $\rho_{1}$ o $\theta=S$ and $\rho_{1}\left[\Pi \overline{w_{l}} . t\right]=$ $\xi$. But $\rho_{1}(t)=\rho_{1}\left(\delta_{1}\left(t_{1}\right)\right)$ by definition of $H_{1}$, and $\rho_{1}\left(\delta_{1}\left(t_{1}\right)\right)=\rho_{1}\left(\theta\left(\theta\left(\delta\left(t_{1}\right)\right)\right)\right)=$ $S\left(\delta_{1}\left(t_{1}\right)\right)$. Thus $\xi=S\left[\Pi \overline{w_{l}} . \delta_{1}\left(t_{1}\right)\right]$. We can therefore rewrite the above instance of the let rule as:

$$
\frac{S[H] \vdash M: S\left[\Pi \overline{w_{l}} . \delta_{1}\left(t_{1}\right)\right] \quad S\left[H, x: \Pi \overline{w_{l}} \delta_{1}\left(t_{1}\right)\right] \vdash N: T}{S[H] \vdash \operatorname{let} x=M \text { in } N: T} \text { let } .
$$

The critical observation is that

$$
\left[H_{e}, x \mapsto \Lambda \overline{v_{m}} \cdot\left(\sigma_{1}, t_{1}\right)\right] \downarrow=\left(\delta_{1},\left[\theta[H], x: \Pi \overline{w_{l}} . \delta_{1}\left(t_{1}\right)\right]\right)
$$

$\overline{{ }^{1} \text { We know } \theta}$ exists since $\delta^{\prime}=$ join $(\delta, \sigma)$. 
But $S[H]=\rho_{1} \circ \theta \circ \theta[H]=S[\theta[H]]$. We can therefore eliminate $\theta$ by absorbing it into $S: S\left[H, x: \Pi \overline{w_{l}} . \delta_{1}\left(t_{1}\right)\right]=S\left[\theta[H], x: \Pi \overline{w_{l}} \delta_{1}\left(t_{1}\right)\right]$. Thus by inductive hypothesis on the second premise we have

$$
W_{\Pi}\left(H_{e}, x \mapsto \Lambda \overline{v_{m}} \cdot\left(\sigma_{1}, t_{1}\right) ; N\right)=\Lambda \overline{u_{n}} \cdot\left(\sigma_{2}, t_{2}\right) .
$$

Let $\left[H_{e}, x \mapsto \lambda \overline{v_{m}} .\left(\sigma_{1}, t_{1}\right), y \mapsto \Lambda \overline{u_{n}} \cdot\left(\sigma_{2}, t_{2}\right)\right] \downarrow=\left(\delta_{2}, H_{2}\right), \theta_{2} \circ \delta_{1}=\delta_{2}$, and $H_{2}(y)=\Pi \overline{z_{k}} \cdot t_{2}$. The inductive hypothesis also gives a $\rho_{2}$ such that $\rho_{2} \circ \theta_{2}=S$ and $\rho_{2}\left[\Pi \overline{z_{k}} \cdot t_{2}\right]=T$.

Now, $j \operatorname{oin}\left(\sigma_{2}, \sigma_{1}\right)=\sigma$ succeeds since $\delta_{2}$ exists $\left(\delta_{2}\right.$ is an instance of $\sigma_{2}$ and $\left.\sigma_{1}\right)$, and so

$$
W_{\Pi}\left(H_{e} ; \text { let } x=M \text { in } N\right)=\Lambda \overline{u_{n}} \Lambda \overline{v_{m}} \cdot\left(\sigma, \sigma\left(t_{2}\right)\right)
$$

succeeds. We also have $\left[H_{e}, y \mapsto \Lambda \overline{u_{n}} \Lambda \overline{v_{m}} \cdot\left(\sigma, \sigma\left(t_{2}\right)\right)\right] \downarrow=\left(\delta_{2}, H_{3}\right)$, and we know that $H_{3}(y)=\Pi \overline{z_{k}} \cdot \delta_{2}\left(t_{2}\right)$. Now $\theta_{2} \circ \theta \circ \delta=\delta_{2}$ and $\rho_{2} \circ\left(\theta_{2} \circ \theta\right)=S \circ \theta=S$. Finally, $\delta_{2}\left(t_{2}\right)=t_{2}$ by definition of $\delta_{2}$, and so

$$
\rho_{2}\left[\Pi \overline{z_{k}} \cdot \delta_{2}\left(t_{2}\right)\right]=\rho_{2}\left[\Pi \overline{z_{k}} \cdot t_{2}\right]=T .
$$

Corollary 6. (Completeness of $W_{\Pi}$ )

For a closed type environment $H$ such that $H \vdash M: T, W_{\Pi}(H \uparrow ; M)=\Lambda \overline{v_{m}} \cdot(\sigma, t)$ such that $T$ is an instance of $\Pi \overline{v_{m}} . t$.

Proof. We may assume, without loss of generality, that $\overline{v_{m}}$ are distinct from all variables in $H$. Set $S=\sigma$. It follows easily from the definition of the algorithm that $\sigma$ does not contain variables other than $\overline{v_{m}}$ in its support. Thus $S[H]=H$. Similarly from the definition of the algorithm, $\sigma(t)=t$. In terms of the above theorem, here $\delta=\emptyset$ and $\delta^{\prime}=\sigma$, so we set $\rho=\emptyset$ and the corollary follows.

The $\downarrow$ operation is not needed in the algorithm for closed type environments since in the returned substitution all fugitives are captured. If the environment can be initially open, then we must free the innocent variables from bondage. The generalized $W_{I I}$ algorithm merely requires a simple extra step: Let $W_{I I}(H \uparrow ; M)=\Lambda \overline{v_{m}} .(\sigma, t)$. Then $\left.\left[H \uparrow, y \mapsto \Lambda \overline{v_{m}} .(\sigma, t)\right]\right\rfloor=\left(\sigma, H^{\prime}\right)$. Return $H^{\prime}(y)$. It will follow that $H^{\prime} \vdash M: H^{\prime}(y)$.

\section{Declarative Implementation}

The eager quantification technique arose from attempts to implement type inference in a higher-order logic programming language. Such a declarative treatment will aid the analysis of functional languages in the context of logical frameworks, such as the dependent-type calculus LF [8]. The desire here is for an executable proof-theoretic formulation of type inference. That is, type inference should be presentable as proof search. The original Damas-Milner calculus is too nondeterministic for this purpose. Previous attempts at its alteration either took 
short-cuts with the let case or were stopped by gen. In [6], Hannan gave prooftheoretic formulations of the natural semantics of ML. But his technique for let was basically to replace let $x=M$ in $N$ with $N[M / x]$. To allow let-expressions to be typed naturally, Harper defined in [7] an "algorithmic" version of the Damas-Milner calculus for the express purpose of allowing the modified typing rules of the new calculus to become logic programs that yield principal type schemes. He defined a predicate called witnessed that captures the maximality condition implemented by gen. Application of the gen operation is replaced by proving that a type scheme is witnessed. Specifying the witnessed predicate directly as logic programming, however, requires a forward-chaining operation which is inconsistent with the goal-directed nature of logic-programming. Another problem with type inference was the need for an inexhaustible supply of new variables. In the context of "meta-programming in logic," one can either use the meta-logic's inherent "logic variables" or define data structures such as strings to represent object-level variables. Using the meta-logic's own variables (called the "non-ground representation") is only adequate for a very small range of problems ${ }^{2}$. Strings and similar structures are too algorithmic and "low level."

It is at this point in the type inferencing algorithm, when "new" variables are needed, that higher-order abstract syntax, combined with a logic programming environment, can be used to advantage. In intuitionistic logic (which forms the basis of many logic programming languages), $\forall x F$ is provable if and only if for a new symbol $a, F[a / x]$ is provable. Thus the process of "creating a new type variable $a$ " can be represented naturally with the intuitionistic quantification $\forall a$. The $\lambda$ clause of the type inference algorithm can be automatically implemented in a logic programming language supporting positive occurrences of $\forall$-quantification. Furthermore, the $\forall$ quantifier is represented in the (meta-level) simply typed $\lambda$-calculus as a second order constant of type $($ term $\rightarrow$ form $) \rightarrow$ form (where term and form classify object-level terms and formulas respectively). The consequence of this is that, although $a$ is supposed to represent a new free variable at the object level, it is in fact represented as a $\lambda$-bound variable at the meta level. That is, at the meta-level of higher-order abstract syntax, all type variables are bound variables. $\lambda$-abstraction immediately enforces the proper scoping of the dynamic "new" variables used in type inference. This uniform treatment of type variables at the meta-level is what allows $\alpha$-conversion to replace the gen operation in allowing for multiple instances of polymorphic types.

A full implementation of the $W_{\Pi}$ algorithm has been given in the logic programming language $L_{\lambda}$ [15] without using any extra-logical extensions. The language of $L_{\lambda}$, which is a simplification of the better known $\lambda$ Prolog, can be directly embedded in a variety of more powerful logical frameworks. This implementation is described in the author's Ph.D. thesis [13].

${ }^{2}$ See $[9,13]$ for further discussion of issues in meta-programming in logic. 


\section{Related Work}

The technique presented here is also related to the work of Leivant [12], Appel and Shao [1] and Jim [10] (among others) in type inferencing with conjunctive types or multi-environments (environments where variables map to sets of types). Leivant's algorithm " $V$ " returns a multi-environment (or multi-base) and a type given a program expression. Type inference in algorithm $V$ does not take place under a given type environment. As a consequence, there is nothing to constrain the generalization of free type variables. Variables can be given multiple instantiations which are then resolved at the end. But algorithm $V$ does not include a case for ML's let. Leivant chose to address let polymorphism in the context of a rank 2 conjunctive type discipline. Wand [18] gave a similar algorithm, which likewise bypassed 1et. Appel and Shao's algorithm $W^{*}$ [1] can be seen as essentially an extension of algorithm $V$ to include let. They use a procedure called Monounify which serves basically the same purpose as join. $W^{*}$ is similar to the approach here in that it too does not use gen ( $g$ en would be meaningless since there is no environment in the input to $W^{*}$ ). Instead, for the let case $W^{*}$ uses an operation called Polyunify, which generates a new set of copies of multi-environments (or "assumption environments") for every occurrence of the let-bound variable. The Polyunify technique is a "brute force" method akin to replacing let $x=M$ in $N$ with $N[M / x]$. The multi-environment returned by $W^{*}$ can be enormous, and will have to be further resolved with a given type environment (using their Match procedure) to derive the final type. Because of this complexity, Appel and Shao themselves favored a customization of Kaes' algorithm " $D$ " [11] for their purpose of smartest recompilation. Furthermore, the correctness of $W^{*}$ was proved by a reduction to the correctness of algorithm $W$, and not to the Damas-Milner typing discipline itself.

The motivation for $W^{*}$ was to support separate compilation, where the types of program variables are not always available. Each program variable is always eagerly given the most general type (a free type variable), and the various possible instantiations are resolved when the type is finally known. The algorithm $W_{\Pi}$ as given already contains the essential components necessary for this purpose. We can assign to each program variable that is not contained in the known type environment the most general type scheme $\Pi v . v$. Then $W_{I}$ will return a substitution containing the different possible instantiations of $v$. For example, assume that the type of $f$ is unknown. Consider the expression let $x=(f 2)$ in $(f 2.5)$. If $f$ is mapped to $\Lambda v \cdot(\emptyset, v)$, then $W_{I I}$ will return the structure

$$
\Lambda b \Lambda c \Lambda v_{1} \Lambda v_{2} \cdot\left(\left[r e a l \rightarrow c / v_{2}, \text { int } \rightarrow b / v_{1}\right], c\right) .
$$

If we knew that the variables $v_{1}$ and $v_{2}$ are in fact copies of the type scheme $\Pi I v . v$, then we can infer the correct type for the expression once the type of $f$ is available. Assume we now know that the type of $f$ is actually $\Pi v . v \rightarrow v$. We can apply Appel and Shao's Match technique to the two instantiations real $\rightarrow c$ and int $\rightarrow b$ with two separate instances of $\Pi v \cdot v \rightarrow v: \Pi u . u \rightarrow u$ and $\Pi w . w \rightarrow w$. This will reveal that $c=$ real and $b=i n t$, and therefore real should be the 
type for let $x=(f 2)$ in $(f 2.5)$. To implement this technique correctly, $W_{\Pi}$ must be modified so that we can identify which variables are copied from type schemes $\Pi v v . v$ associated with undeclared program variables. One approach is to label these special type variables with the program variable they are associated with. This approach would be similar to Appel and Shao's adaptation of Kaes' algorithm $D$ for constrained types [11]. However, algorithm $D$ again uses the gen operation in the let case.

The purpose of the above discussion is to clarify the relationship between our algorithm and work in conjunctive types. It is not our immediate aim here to formulate an algorithm in a conjunctive type discipline. We wish to derive principal types as in ML, and not principal typings (as in [10]). Instead, we use the technique of conjunctive types at an intermediate level (when multiple substitutions are kept inside extended environments) in order to facilitate the typing of let-expressions.

\section{Conclusion and Future Work}

The traditional gen operation is incompatible with a declarative, logical framework approach to formulating principal type inference. It is hoped that our new approach will provide a starting point from which various issues of type inference can be studied in declarative settings, without ignoring let-polymorphism. It of course remains to extend $W_{\Pi}$ to other language constructs. We also hope to study, in the context of the eager quantification technique, type disciplines other than ML polymorphism (in particular principal typings and conjunctive types). This will lead to, for example, the use of our technique with respect to polymorphic references. It is hoped that we will be able to accept more typesafe programs than current methods. The $W_{\Pi}$ algorithm can also lead to the early reportage of typing errors. Because substitutions are composed instead of joined in algorithm $W$, by the time we discover a type error the substitutions may have obscured its origin. Combined with a constrained typing discipline, the $W_{I I}$ technique can potentially offer a new solution to this problem.

\section{Acknowledgments}

Much of this research was conducted under the supervision and support of Dale Miller at the University of Pennsylvania. The author also wishes to thank Sandip Biswas for invaluable help in preparing this paper.

\section{References}

1. Andrew Appel and Zhong Shao. Smartest Recompilation. In Tenth Annual ACM SIGPLAN-SIGACT Symposium on Principles of Programming Languages, January 1993. Longer version as Princeton University Technical Report CS-TR-39592. 
2. Luis Damas and Robin Milner. Principal type-schemes for functional programs. In Ninth Annual ACM SIGPLAN-SIGACT Symposium on Principles of Programming Languages, pages 207-212, January 1982.

3. Scott Dietzen and Frank Pfenning. A declarative alternative to assert in logic programming. In Proceedings of the 1991 International Logic Programming Symposium, pages 372-386. MIT Press, 1991.

4. G. Dowek et al. The Coq proof assistant user's guide. Technical Report 134, INRIA, 1993.

5. C. A. Gunter. Semantics of Programming Languages: Structures and Techniques. Foundations of Computing. MIT Press, 1992.

6. John Hannan. Extended natural semantics. Journal of Functional Programming, 3(2):123-152, April 1993.

7. Robert Harper. Systems of polymorphic type assignment in LF. Technical Report CMU-CS-90-144, Carnegie Mellon University, Pittsburgh, Pennsylvania, June 1990.

8. Robert Harper, Furio Honsell, and Gordon Plotkin. A framework for defining logics. Journal of the ACM, 40(1):143-184, 1993.

9. P. M. Hill and J. G. Gallagher. Meta-programming in logic programming. Technical Report Report 94.22, University of Leeds, hill@scs.leeds.ac.uk, August 1994. To appear in Vol. 5 of the Handbook of Logic in Artificial Intelligence and Logic Programming, Oxford University Press.

10. Trevor Jim. What are principal typings and what are they good for? Technical Report MIT/LCS TM-532, MIT, November 1995. Extended version of a paper appearing in ACM Symposium on Principles of Programming Languages, 1996.

11. Stefan Kaes. Type Inference in the presence of Overloading, Subtyping, and Recursive types. In 1992 ACM conference on LISP and Functional Programming, San Francisco, CA, pages 193-204. ACM Press, 1992.

12. Daniel Leivant. Polymorphic type inference. In Conference Record of the Tenth Annual ACM Symposium on Principles of Programming Languages, pages 88-98, 1983.

13. Chuck Liang. Substitution, Unification and Generalization in Meta-Logic. PhD thesis, University of Pennsylvania, September 1995.

14. Dale Miller. Abstractions in logic programming. In Piergiorgio Odifreddi, editor, Logic and Computer Science, pages 329-359. Academic Press, 1990.

15. Dale Miller. A logic programming language with lambda-abstraction, function variables, and simple unification. Journal of Logic and Computation, 1(4):497$536,1991$.

16. Lawrence C. Paulson. The foundation of a generic theorem prover. Journal of Automated Reasoning, 5:363-397, September 1989.

17. Frank Pfenning and Conal Elliot. Higher-order abstract syntax. In Proceedings of the ACM-SIGPLAN Conference on Programming Language Design and Implementation, pages 199-208. ACM Press, June 1988.

18. Mitchell Wand. A simple algorithm and proof for type inference. Fundamenta Infomaticae, 10:115-122, 1987. 